\title{
Ekmeklik Buğday Genotiplerinde Geç Kuraklığın Vejetatif Dönem ve Tane Dolum Süresine Etkisi
}

\author{
*Murat AYDIN $\quad$ Ali ÖZTÜRK \\ Atatürk Üniversitesi Ziraat Fakültesi, Tarla Bitkileri Bölümü, Erzurum \\ *Sorumlu yazar e-posta (Corresponding author; e-mail): maydin@atauni.edu.tr
}

\begin{abstract}
Öz
Vejetatif dönem fotosentez alanının büyüklüğü ve potansiyel tane sayısını, tane dolum süresi ise tane ağırlığını etkilediğinden tane verimi ile yakından ilişkilidir. Geç kuraklığın bu karakterler üzerindeki etkisinin incelendiği bu araştırma, 2011-2012 ve 2012-2013 ürün yıllarında 64 ekmeklik buğday genotipi ile 8x8 kare latis deneme deseninde, iki ayrı (sulu koşullar, geç kuraklık stresi) deneme halinde Erzurum koşullarında yürütülmüştür. Ürün yıllarının ortalamasına göre, genotiplerin sulu koşullardaki vejetatif dönemleri (1 Haziran=1) 12.1 gün (Bolal 2973)-19.8 gün (Lancer); geç kuraklık koşullarındaki vejetatif dönemleri ise 11.9 gün (Prostor)-19.1 gün (Gün 91) arasında değişmiştir. Ürün yılları ve genotiplerin ortalamasına göre, vejetatif dönem sulu koşullarda 16.7 gün, geç kuraklık koşullarında ise 16.0 gün olmuştur. Genotiplerin tane dolum süresi, sulu koşullarda 34.9 gün (Pehlivan)-41.4 gün (Palandöken 97); geç kuraklık koşullarında ise 27.2 gün (Kırkpınar 79)-32.5 gün (Türkmen) arasında değişim göstermiştir. Tane dolum süresi sulu koşullarda 38.1 gün iken, geç kuraklık koşullarında kısalarak 29.1 gün olmuştur. Geç kuraklık koşullarında tane dolum süresindeki kısalmanın en fazla Özlü Buğday, Ankara 093/44, Zerin, Köse 220/39 ve Koca Buğday; en az ise Mızrak, Alparslan, Gün 91, Türkmen ve Aytın 98 genotiplerinde olduğu belirlenmiştir.
\end{abstract}

Anahtar Kelimeler: Buğday, kuraklık, vejetatif dönem, tane dolum süresi

\section{Effect of Late Drought on Vegetative Period and Grain Filling Period in Bread Wheat Genotypes}

\begin{abstract}
Vegetative period (VP) and grain filling period (GFP) are closely related to grain yield since VP affects size of photosynthetic area and the number of potential grain, while GFP affects grain weight. To investigate the effects of late drought on these characters, this research carried out with 64 bread wheat genotypes under Erzurum conditions in 2011-12 and 2012-13 cropping seasons according to the 8x8 square lattice experimental design in two experiments (irrigated and late drought stress). According to average of cropping seasons, VP of genotypes ranged from 12.1 days (Bolal 2973) to 19.8 days (Lancer) under irrigated condition, while their VP varied between 11.9 days (Prostar) and 19.1 days (Gün 91) in late drought condition. According to the average of the crop year and genotypes, VP was 16.7 days in irrigated condition and was 16.0 days in the late drought condition. GFP of genotypes varied between 34.9 days (Pehlivan) and 41.4 days (Palandöken 97) in irrigated condition, while their GFP ranged from 27.2 days (Kırkpınar 79) to 32.5 days (Türkemen). GFP was 38.1 days in irrigated condition, while it was 29.1 days in late drought condition. The maximum reduction in GFP was determined in Özlü Buğday, Ankara 093/44, Zerin, Köse 220/39 and Koca Buğday genotypes, while minimum reduction was determined in Mızrak, Alparslan, Gün 91, Türkmen and Aytın 98 genotypes in late drought condition.
\end{abstract}

Keywords: Wheat, drought, vegetative period, grain filling period

\section{Giriş}

B uğdayın tane verimi büyük ölçüde vejetatif dönemde belirlenen depo kapasitesine ve tane dolum süresindeki fotosentez kapasitesine bağlıdır. Buğday tanesinde biriken asimilatların büyük bir kısmı tane dolum dönemindeki fotosentezden kaynaklandığından fotosentez organlarının bu dönemdeki büyüklüğü ve ömrü verimle ilgilidir. Konu ile ilgili araştırmalarda tane dolum süresi ile tane verimi (Gebeyehou et al. 1982; Öztürk ve Akten 1999; Tiwari 2007), başaktaki tane sayısı (Öztürk ve Akkaya 1996; Öztürk ve Akten 1999) ve bin tane ağırlığ 
(Öztürk ve Akkaya 1996) arasında olumlu ve önemli ilişki olduğu belirlenmiştir. Bu nedenle tane dolum süresinin uzun olması tahıllarda istenilen bir özelliktir (Nass and Reiser 1975).

Vejatatif dönem ve tane dolum süresi genetik yapı, ekolojik koşullar ve yetiştirme tekniklerinin kontrolü altındadır (Öztürk ve Çağlar 1999). Bu karakterler yönünden buğday genotipleri arasında önemli farklar vardır (Bruckner and Frohberg, 1987; Çağlar ve ark. 2006). Ancak, tane dolum süresinin kalıtım derecesi düşük olup (Egli 1998), çevre koşullarından önemli ölçüde etkilenmektedir (Öztürk ve Akten, 1999; Sanjari et al. 2011). Kuraklık stresi, bitki büyüme ve gelişimini etkileyen önemli çevresel faktörlerden biridir. Bu stres faktörü altında yetişen buğdayın yaşam döngüsü normal şartlara göre daha hızlı tamamlanmaktadır. Sonuç olarak, yaşam döngüsü boyunca daha az asimilat birikimi gerçekleşmekte ve dolaysıyla biyokütle üretimi daha az olmaktadır (Fischer and Maurer 1976; Wahid et al. 2007). Kuraklık buğday gelişiminin farklı dönemlerinde etkili olmakla birlikte, çiçeklenme ve tane dolum dönemi kuraklık stresine özellikle duyarlıdır (Prasad et al. 2011). Kuraklık, ülkemizde genellikle buğdayın çiçeklenme dönemine yakın başlamakta ve tane dolum döneminde etkisini artırmaktadır (Öztürk ve Çağlar, 1999). Tane dolumunum erken dönemindeki su stresi yaprak yaşlanmasını hızlandırarak fotosentezi (Farooq et al. 2014) ve kuru madde birikimi için depo kapasitesini azaltır (Nicolas 1985). Verimdeki azalma temel olarak, kuraklığın başak oluşumu ve çiçeklenme sonrası yaprak alanı üzerindeki olumsuz etkisinden kaynaklanır (Öztürk 1999). Bu araştırmada, geç kuraklığın ekmeklik buğday genotiplerinde vejetatif dönem ve tane dolum süresine olan etkisi incelenmiştir.

\section{Materyal ve Yöntem}

Araştırma, Atatürk Üniversitesi Ziraat Fakültesi Tarımsal Araştırma Merkezi'nin 4 nolu deneme alanında, 2011-2012 ve 2012-2013 ürün yıllarında iki ayrı deneme halinde yürütülmüştür. Denemede bitki materyali olarak toplam 64 ekmeklik buğday genotipi kullanıımıştır.

Deneme I (sulu koşullar): Topraktaki elverişli suyun yaklaşık \%50'si tüketildiğinde bütün parseller yüzey sulama yöntemi ile sulanmıştır.

Deneme II (geç kuraklık stresi): Bitkiler gebecik dönemi başlangıcına kadar doğal yağış koşullarında yetiştirilmiş, genotiplerin \%50'si gebecik dönemi başlangıcına ulaştığı 29 Mayıs 2012 ve 24 Mayıs 2013 tarihlerinde deneme alanının üzeri polietilen örtü ile kapatılmış, bitkilerin bu tarihlerden hasada kadar düşen yağışları (2011-12 ve 2012-13 ürün yıllarında sırasıyla $49.6 \mathrm{~mm}$ ve $42.1 \mathrm{~mm}$ ) alması engellenmiştir.

Deneme yeri topraklarının tekstür sınıfı killitın, organik madde ve azot oranları az, reaksiyonu hafif alkali, az kireçli, fosfor yönünden yeterli, potasyum yönünden ise çok zengindir. Araştırma yerinin yıllık toplam yağış miktarı ve ortalama sıcaklığı uzun yıllar ortalamasına göre sırasıyla $398.8 \mathrm{~mm}$ ve $5.0^{\circ} \mathrm{C}$ 'dir. Bu değerler, 2011-12 ürün yılında sırasılyla $250.5 \mathrm{~mm}$ ve $3.9^{\circ} \mathrm{C}$ iken, $2012-2013$ ürün yılında $311.1 \mathrm{~mm}$ ve $6.4^{\circ} \mathrm{C}$ olmuştur. Toprakların $60 \mathrm{~cm}$ derinliğindeki hacimsel nem içeriği sapa kalkma başlangıcından fizyolojik olgunluk dönemine kadar 7 gün aralıkla toprak nemölçer (GMS, Watermark Soil Moisture Sensor Model 200SS) ile ölçülmüştür. Toprak hacimsel nem içeriği sulu koşullarda 2011-12 ve 2012-13 ürün yıllarında sırasıyla \%29.1-40.1 ve \%28.0-38.1 arasında değişmiştir. Geç kuraklık koşullarında, 2011-12 ve 2012-13 ürün yıllarının ilk ölçüm tarihlerinde toprak hacimsel nem içeriği sırasıyla \%40.2 ve \%38.2 iken, bu tarihlerden sonra düzenli şekilde azalarak son ölçüm tarihlerinde sırasıyla \%18.6 ve $\% 14.1$ olmuş ve solma noktasının altına inmiştir. Denemeler nadas araziye kurulmuş, nadas yıllarında (201011 ve 2011-12 ürün yıllarında sırasıyla $513.3 \mathrm{~mm}$ ve $250.5 \mathrm{~mm}$ ) ve ürün yıllarında düşen yağışlar ve toprak nem ölçüm değerleri dikkate alındığında, 2012-13 ürün yılı 2011-12 ürün yılına göre daha kurak geçmiştir. Denemeler 8x8 kare latis deneme deseninde ve 2 tekerrürlü olarak yürütülmüştür. Toprak hazırlığı yapılmış nadas araziye Eylül ayı içerisinde, Deneme l'de parsel mibzeri ile Deneme Il'de ise elle ve $\mathrm{m}^{2} \mathrm{ye}$ 475 canlı tohum sıklığında ekim yapılmıştır. Deneme l'de her parsel $1.2 \mathrm{~m} \times 6.0 \mathrm{~m}$ ebatlarında olmak üzere, $20 \mathrm{~cm}$ aralıkla 6 bitki sırası içermiştir. Deneme Il'de genotipler $1.5 \mathrm{~m}$ uzunluğunda, $4-5 \mathrm{~cm}$ derinliğinde ve $20 \mathrm{~cm}$ aralıkla açılan markör sıralarına 2 sıra halinde ekilmiştir. Parseller $6 \mathrm{~kg} / \mathrm{da} \mathrm{N}$ ve $5 \mathrm{~kg} / \mathrm{da} \mathrm{P}_{2} \mathrm{O}_{5}$ olacak şekilde gübrelenmiş, fosforun tamamı ve azotun yarısı ekimle birlikte, azotun diğer yarısı ise sapa kalkma başlangıcında uygulanmıştır. 1 Haziran tarihinden, çiçeklenme tarihine (parseldeki başakların yaklaşık \%50'sinde orta kısımdaki çiçeklerde anterlerin dışarı çıkış zamanı) kadar geçen gün sayısı vejetatif dönem (gün); çiçeklenme tarihinden fizyolojik olgunluğa 
(parseldeki başakların yaklaşık \%50'sinde yeşil rengin tamamen kaybolduğu gün) kadar geçen gün sayısı ise tane dolum süresi (gün) olarak kabul edilmiştir. Veriler deneme planına uygun olarak MSTAT-C bilgisayar programı ile varyans analizine tabi tutulmuş, genotiplere ait ortalamaların karşılaştırılmasında önemlilik düzeyine göre Duncan çoklu karşılaştırma testi kullanılmıştır. Ürün yıllarının ayrı analizlerinde, tesadüf blokları deneme deseni ile karşılaştırıldığında, latis etkinliği \%100'den daha yüksek olduğu için düzeltilmiş değerler verilmiştir. Ürün yıllarının birlikte analizleri, karışık modelde varyans analizine tabi tutulmuş (IBM SPSS Statistics 20), modelde yıl ve tekerrür rastgele etki olarak kabul edilmiş ve düzeltilmiş değerler sunulmuştur.

\section{Bulgular ve Tartışma}

\section{Vejetatif Dönem}

Vejetatif dönem yönünden genotipler arasındaki farklar ile ürün yılları arasındaki fark ve genotip $x$ yıl interaksiyonu iki deneme koşulunda da önemli olmuştur (Çizelge 1). Genotiplerin sulu koşullardaki vejetatif dönemleri 2011-12 ürün yılında 14.2-22.7 gün, 2012-13 ürün yılında 10.3-17.3 gün, yılların ortalaması olarak ise 12.1-19.8 gün arasında değişmiştir. Genotiplerin geç kuraklık stresi koşullarındaki vejetatif dönemleri ise 2011-12 ürün yılında 14.3-22.7 gün, 2012-13 ürün yılında 9.8-15.8 gün, yılların ortalaması olarak ise 11.9-19.1 gün arasında olmuştur. Bu sonuçlar, genotiplerin çiçeklenme tarihleri arasında sulu koşullarda 7.0-8.5 gün, geç kuraklık stresi koşullarında ise 6.0-8.4 gün fark olduğunu göstermektedir. Buğday genotiplerinin vejetatif dönem yönünden önemli derecede farklı olduklarına dikkat çeken araştırıcılar, bulgularımızla yakın olarak genotiplerin çiçeklenme tarihleri arasında 8-11 gün (Wiegand et al. 1981), 6-9 gün (Öztürk ve Akkaya 1996) veya 8.4 gün (Çağlar ve ark. 2006) fark bulmuşlardır. Sulu koşullarda en kısa vejetatif dönem birinci ürün yılında Bolal 2973, Alparslan ve Prostor; ikinci ürün yılında Bolal 2973, Aksel 2000 ve Aldane genotiplerinde, en uzun vejetatif dönem ise ilk ürün yılında Lancer, Uzunyayla ve Kutluk 94; ikinci ürün yılında Tir, Yayla 305 ve Özlü Buğday genotiplerinde saptanmıştır. Geç kuraklık stresi koşullarında en kısa vejetatif döneme birinci ürün yılında Alparslan, Bolal 2973 ve Prostor; ikinci ürün yılında Prostor, Aldane ve Alparslan genotipleri, en uzun vejetatif döneme ise ilk ürün yılında Lancer, Gün 91 ve Uzunyayla; ikinci ürün yılında Sürak 1593/51, Altay 2000 ve Gün 91 genotipleri sahip olmuştur (Çizelge 1). Bitkilerin kışa girdikleri gelişme dönemi, kar örtüsü kalktıktan sonra bitkilerin ilkbaharda yeniden büyümeye başlama zamanı ve takip eden süreçteki sıcaklık ve nem koşullarına bağlı olarak vejetatif dönem ürün yıllarına göre farklılık gösterebilir. İki denemede de, daha düşük toprak nem içeriği nedeniyle, vejetatif dönem ikinci ürün yılında birinci ürün yılına göre önemli derecede kısalmıştır. Ürün yılları ve genotipler ortalamasına göre, vejetatif dönem sulu koşullarda 16.7 gün, geç kuraklık stresi koşullarında ise 16.0 gün olmuştur. Çiçeklenmeye yakın dönemde toprakta yeterli nem bulunması nedeniyle, örtü uygulaması ile çiçeklenme tarihi arasındaki sürede (20-30 gün) engellenen yağışlar vejetatif dönemi büyük ölçüde değiştirmemiştir.

\section{Tane Dolum Süresi}

Tane dolum süresi yönünden genotipler arasındaki farklar ile ürün yılları arasındaki fark ve genotip $x$ yıl interaksiyonu iki denemede de önemli olmuştur. Genotiplerin sulu koşullardaki tane dolum süreleri 2011-12 ürün yılında 39.348.9 gün, 2012-13 ürün yılında 28.4-35.1 gün, yılların ortalaması olarak ise 34.9-41.4 gün arasında değişmiştir. Genotiplerin geç kuraklık stresi koşullarındaki tane dolum süreleri ise 2011-12 ürün yılında 30.6-39.9 gün, 2012-13 ürün yılında 19.8-29.8 gün, yılların ortalaması olarak ise 27.2-32.5 gün arasında gözlenmiştir.

Sulu koşullarda en uzun tane dolum süresi ilk yılda Zerin, Bayraktar 2000 ve Karasu 90; ikinci yılda Prostor, Sultan 95 ve Palandöken 97 genotiplerinde, en kısa tane dolum süresi ise ilk yılda Mızrak, Alparslan ve Pehlivan; ikinci yılda Kırkpınar 79, Aytın 98 ve Doğu 88 çeşitlerinde tespit edilmiştir.

Geç kuraklık stresi koşullarında en uzun tane dolum süresine birinci yıl Palandöken 97, Bayraktar 2000 ve Bezostaja 1; ikinci yıl Dağdaş 94, Tükmen ve Mızrak çeşitleri, en kısa tane dolum süresine ise ilk yıl Yakar 99, Kutluk 94 ve Gün 91; ikinci yıl Polatlı Kösesi, Sürak 1593/51 ve Koca Buğday genotipleri sahip olmuştur (Çizelge 2). Diğer araştırmalarda da tane dolum süresi yönünden buğday genotipleri arasında önemli farklar bulunmuş, bu sürenin 33.7-36.4 gün (Hunt et al. 1991) veya 34.9-39.3 gün (Çağlar ve ark. 2006) arasında olduğu belirlenmiştir. Tane dolum süresi, tanelere biriken asimilat miktarını etkilediğinden tane ağırlığı ve tane verimi ile olumlu ilişkilidir (Öztürk ve Akten 1999). 
Table 1. Buğday genotiplerinin vejetatif dönemleri (gün, 1 Haziran=1)

Table 1. Vegetative periods of wheat genotypes (day, 1 June $=1$ )

\begin{tabular}{|c|c|c|c|c|c|c|c|}
\hline \multirow{2}{*}{ No } & \multirow{2}{*}{ Genotipler } & \multicolumn{3}{|c|}{ Sulu koşullar } & \multicolumn{3}{|c|}{ Geç kuraklık } \\
\hline & & $2011-12$ & $2012-13$ & Birleşik & $2011-12$ & $2012-13$ & Birleşik \\
\hline 1 & Aksel 2000 & $15.2 \mathrm{l}-\mathrm{p}$ & $10.4 p$ & $12.6 \mathrm{uv}$ & $15.1 \mathrm{i}-\mathrm{m}$ & $11.5 \mathrm{~h}-\mathrm{m}$ & $13.3 \mathrm{r}-\mathrm{u}$ \\
\hline 2 & Aldane & 16.6 h-p & $10.5 p$ & $13.5 \mathrm{~s}-\mathrm{v}$ & $15.8 \mathrm{~h}-\mathrm{m}$ & $9.9 \mathrm{Im}$ & $12.8 \mathrm{stu}$ \\
\hline 3 & Alparslan & 14.7 op & 11.7 op & 13.2 tuv & $14.3 \mathrm{~m}$ & $10.4 \mathrm{klm}$ & $12.4 \mathrm{tu}$ \\
\hline 4 & Altay 2000 & $18.3 \mathrm{~d}-\mathrm{m}$ & $14.8 \mathrm{a}-\mathrm{m}$ & $16.5 \mathrm{f}-\mathrm{p}$ & $17.7 \mathrm{~d}-\mathrm{k}$ & $15.6 \mathrm{ab}$ & $16.7 \mathrm{c}-\mathrm{j}$ \\
\hline 5 & Atlı 2002 & $18.1 \mathrm{~d}-\mathrm{m}$ & 14.7 a-n & $16.3 h-p$ & $18.2 \mathrm{~b}-\mathrm{j}$ & $15.1 \mathrm{abc}$ & $16.6 \mathrm{c}-\mathrm{j}$ \\
\hline 6 & Aytın 98 & 19.6 a-h & $15.1 \mathrm{a}-\mathrm{I}$ & $17.3 \mathrm{~b}-\mathrm{m}$ & $19.1 \mathrm{~b}-\mathrm{h}$ & 13.6 a-h & $16.4 \mathrm{c}-\mathrm{I}$ \\
\hline 7 & Bağcı 2002 & $18.7 \mathrm{c}-\mathrm{j}$ & $14.4 c-n$ & $16.5 f-p$ & $18.1 \mathrm{~b}-\mathrm{j}$ & $13.0 \mathrm{c}$-I & $15.5 \mathrm{~h}-\mathrm{q}$ \\
\hline 8 & Bayraktar 2000 & $15.1 \mathrm{~m}-\mathrm{p}$ & $13.5 \mathrm{i}-\mathrm{o}$ & $14.3 q-u$ & $14.7 \mathrm{klm}$ & $10.6 \mathrm{j}-\mathrm{m}$ & 12.7 stu \\
\hline 9 & Bolal 2973 & $14.2 \mathrm{p}$ & $10.3 p$ & $12.1 \mathrm{v}$ & $14.4 \mathrm{Im}$ & $12.0 \mathrm{~g}-\mathrm{I}$ & $13.2 \mathrm{r}-\mathrm{u}$ \\
\hline 10 & Bereket & $17.6 \mathrm{f}-\mathrm{o}$ & $12.9 \mathrm{I}-\mathrm{O}$ & 15.2 o-s & $17.0 \mathrm{f}-\mathrm{m}$ & 12.4 e-k & $14.7 \mathrm{I}-\mathrm{r}$ \\
\hline 11 & Çetinel 2000 & $20.2 \mathrm{a}-\mathrm{g}$ & 14.6 b-n & 17.4 b-I & 20.6 a-e & 14.9 a-d & $17.8 \mathrm{a}-\mathrm{e}$ \\
\hline 12 & Dağdaş 94 & $18.3 \mathrm{~d}-\mathrm{m}$ & $15.2 \mathrm{a}-\mathrm{I}$ & $16.7 \mathrm{f}-\mathrm{o}$ & $18.0 \mathrm{c}-\mathrm{j}$ & $14.1 \mathrm{a}-\mathrm{g}$ & $16.1 \mathrm{e}-\mathrm{m}$ \\
\hline 13 & Demir 2000 & 19.6 a-h & $15.1 \mathrm{a}-\mathrm{I}$ & $17.3 \mathrm{~b}-\mathrm{n}$ & 20.0 a-f & 14.6 a-e & $17.3 \mathrm{~b}-\mathrm{h}$ \\
\hline 14 & Doğu 88 & 19.6 a-h & $15.0 \mathrm{a}-\mathrm{I}$ & $17.3 \mathrm{~b}-\mathrm{n}$ & 20.8 a-e & $15.1 \mathrm{abc}$ & $18.1 \mathrm{a}-\mathrm{d}$ \\
\hline 15 & ES 26 & $18.2 \mathrm{~d}-\mathrm{m}$ & $13.8 \mathrm{f}-\mathrm{o}$ & $16.0 \mathrm{j}-\mathrm{q}$ & $18.4 \mathrm{~b}-\mathrm{i}$ & $15.0 \mathrm{abc}$ & $16.7 \mathrm{c}-\mathrm{j}$ \\
\hline 16 & Gerek 79 & $18.6 \mathrm{c}-\mathrm{j}$ & $14.4 c-n$ & $16.5 \mathrm{f}-\mathrm{p}$ & $18.0 \mathrm{c}-\mathrm{j}$ & $14.1 \mathrm{a}-\mathrm{g}$ & $16.1 \mathrm{e}-\mathrm{m}$ \\
\hline 17 & Gün 91 & 20.9 a-e & $15.2 \mathrm{a}-\mathrm{I}$ & $18.0 \mathrm{a}$ & $22.5 \mathrm{a}$ & $15.5 \mathrm{ab}$ & $19.1 \mathrm{a}$ \\
\hline 18 & Harmankaya 99 & $18.3 \mathrm{~d}-\mathrm{m}$ & $12.4 \mathrm{~m}-\mathrm{p}$ & $15.4 \mathrm{n}-\mathrm{r}$ & $17.1 \mathrm{f}-\mathrm{m}$ & $11.0 \mathrm{I}-\mathrm{m}$ & $14.10-t$ \\
\hline 19 & İkizce 96 & 17.9 e-n & $15.0 \mathrm{a}-1$ & $16.6 \mathrm{f}-\mathrm{p}$ & $17.2 \mathrm{f}-\mathrm{m}$ & $14.0 \mathrm{a}-\mathrm{g}$ & $15.7 \mathrm{~h}-\mathrm{p}$ \\
\hline 20 & İzgi 2001 & $19.0 \mathrm{c}-\mathrm{i}$ & $13.6 \mathrm{~g}-\mathrm{o}$ & $16.4 \mathrm{~g}-\mathrm{p}$ & $18.1 \mathrm{~b}-\mathrm{j}$ & $12.6 \mathrm{~d}-\mathrm{j}$ & $15.5 \mathrm{~h}-\mathrm{q}$ \\
\hline 21 & Karahan 99 & $19.3 \mathrm{~b}-\mathrm{h}$ & $15.0 \mathrm{a}-\mathrm{I}$ & $17.2 \mathrm{~b}-\mathrm{n}$ & $17.1 \mathrm{f}-\mathrm{m}$ & $12.6 \mathrm{e}-\mathrm{j}$ & $14.9 \mathrm{j}-\mathrm{r}$ \\
\hline 22 & Kate A-1 & $17.8 \mathrm{e}-\mathrm{o}$ & $12.9 \mathrm{l}-\mathrm{O}$ & $15.4 \mathrm{~m}-\mathrm{r}$ & $16.9 \mathrm{f}-\mathrm{m}$ & $12.2 \mathrm{f}-\mathrm{k}$ & $14.7 \mathrm{k}-\mathrm{r}$ \\
\hline 23 & Kıraç 66 & 20.9 a-e & $16.2 \mathrm{a}-\mathrm{h}$ & 18.7 a-e & $21.0 \mathrm{a}-\mathrm{d}$ & $14.1 \mathrm{a}-\mathrm{g}$ & $17.6 \mathrm{a}-\mathrm{g}$ \\
\hline 24 & Kırgiz 95 & $18.3 \mathrm{~d}-\mathrm{I}$ & $15.8 \mathrm{a}-\mathrm{k}$ & $17.2 \mathrm{~b}-\mathrm{n}$ & $18.1 \mathrm{~b}-\mathrm{j}$ & $14.1 \mathrm{a}-\mathrm{g}$ & $16.3 \mathrm{~d}-\mathrm{m}$ \\
\hline 25 & Kırik & $19.6 \mathrm{a}-\mathrm{h}$ & 14.9 a-m & $17.2 \mathrm{~b}-\mathrm{n}$ & 20.0 a-f & $14.0 \mathrm{a}-\mathrm{g}$ & $17.1 \mathrm{~b}-\mathrm{I}$ \\
\hline 26 & Kutluk 94 & $21.6 \mathrm{abc}$ & $16.0 \mathrm{a}-\mathrm{k}$ & $18.9 \mathrm{ab}$ & 20.6 a-e & $13.4 b-h$ & $17.1 \mathrm{~b}-\mathrm{I}$ \\
\hline 27 & Lancer & $22.7 \mathrm{a}$ & 16.6 a-e & $19.8 \mathrm{a}$ & $22.7 \mathrm{a}$ & 14.4 a-f & $18.7 \mathrm{ab}$ \\
\hline 28 & Mızrak & $17.3 \mathrm{~g}-\mathrm{o}$ & $14.3 \mathrm{c}-\mathrm{n}$ & $15.9 \mathrm{k}-\mathrm{q}$ & $17.1 \mathrm{f}-\mathrm{m}$ & $13.1 \mathrm{c}$-I & $15.3 \mathrm{I}-\mathrm{q}$ \\
\hline 29 & Müfitbey & $19.6 \mathrm{a}-\mathrm{h}$ & 16.2 a-I & 17.9 b-I & 20.1 a-f & $15.1 \mathrm{abc}$ & 17.7 a-f \\
\hline 30 & Nacibey & $18.6 \mathrm{c}-\mathrm{k}$ & $15.1 \mathrm{a}-\mathrm{I}$ & $16.9 \mathrm{~d}-\mathrm{o}$ & $18.9 \mathrm{~b}-\mathrm{h}$ & 14.6 a-e & $17.0 \mathrm{~b}-\mathrm{I}$ \\
\hline 31 & Nenehatun & $17.7 \mathrm{e}-\mathrm{o}$ & 16.4 a-f & 17.1 b-n & $18.9 \mathrm{~b}-\mathrm{h}$ & $14.0 \mathrm{a}-\mathrm{g}$ & $16.6 \mathrm{c}-\mathrm{j}$ \\
\hline 32 & Palandöken 97 & 15.6 j-p & 15.5 a-I & $15.7 \mathrm{l}-\mathrm{r}$ & $15.1 \mathrm{j}-\mathrm{m}$ & $13.6 \mathrm{a}-\mathrm{h}$ & $14.5 \mathrm{~m}-\mathrm{r}$ \\
\hline 33 & Pehlivan & 15.4 k-p & 12.2 nop & $13.7 \mathrm{~s}-\mathrm{v}$ & 15.8 h-m & $12.9 \mathrm{c}$ - I & 14.3 n-s \\
\hline 34 & Prostor & 14.8 nop & $13.3 \mathrm{k}-\mathrm{o}$ & $14.1 \mathrm{r}-\mathrm{u}$ & $14.4 \mathrm{Im}$ & $9.8 \mathrm{~m}$ & $11.9 \mathrm{u}$ \\
\hline 35 & Soyer02 & $18.4 \mathrm{c}-\mathrm{k}$ & 14.0 e-o & 16.2 I-p & $18.4 \mathrm{~b}-\mathrm{h}$ & $13.3 b-h$ & $15.8 \mathrm{f}-\mathrm{o}$ \\
\hline 36 & Sönmez 2001 & 16.5 h-p & $13.6 \mathrm{~h}-\mathrm{o}$ & 15.1 o-s & $16.4 \mathrm{~g}-\mathrm{m}$ & 11.5 h-m & $13.9 \mathrm{q}-\mathrm{t}$ \\
\hline 37 & Sultan 95 & $18.4 \mathrm{c}-\mathrm{k}$ & $15.5 \mathrm{a}-\mathrm{I}$ & $16.8 \mathrm{~d}-\mathrm{o}$ & 19.9 a-f & $15.0 \mathrm{abc}$ & $17.3 \mathrm{~b}-\mathrm{h}$ \\
\hline 38 & Süzen 97 & $17.3 \mathrm{~g}-\mathrm{o}$ & 16.4 a-f & $16.8 \mathrm{~d}-\mathrm{o}$ & $17.7 \mathrm{~d}-\mathrm{k}$ & $15.0 \mathrm{abc}$ & $16.3 \mathrm{c}-\mathrm{I}$ \\
\hline 39 & Tosunbey & $16.0 \mathrm{i}-\mathrm{p}$ & $13.7 \mathrm{f}-\mathrm{o}$ & $14.8 p-t$ & $16.2 \mathrm{~g}-\mathrm{m}$ & $11.9 \mathrm{~g}-\mathrm{I}$ & $14.0 \mathrm{p}-\mathrm{t}$ \\
\hline 40 & Türkmen & $17.8 \mathrm{e}-\mathrm{o}$ & $15.8 a-k$ & $16.8 \mathrm{~d}-\mathrm{o}$ & $18.9 \mathrm{~b}-\mathrm{h}$ & $14.0 \mathrm{a}-\mathrm{g}$ & $16.4 \mathrm{c}-\mathrm{I}$ \\
\hline 41 & Uzunyayla & $22.3 \mathrm{ab}$ & $15.3 \mathrm{a}-\mathrm{I}$ & 18.7 a-d & $22.4 \mathrm{a}$ & $14.9 \mathrm{abc}$ & $18.7 \mathrm{ab}$ \\
\hline 42 & Yakar 99 & $18.2 \mathrm{~d}-\mathrm{m}$ & $13.5 \mathrm{j}-\mathrm{o}$ & $15.9 \mathrm{k}-\mathrm{q}$ & $17.5 \mathrm{e}-\mathrm{I}$ & 12.4 e-k & $14.9 \mathrm{j}-\mathrm{r}$ \\
\hline 43 & Zencirci 2002 & $18.8 \mathrm{c}-\mathrm{j}$ & $14.1 \mathrm{~d}-\mathrm{o}$ & $16.5 \mathrm{f}-\mathrm{p}$ & $19.1 \mathrm{~b}-\mathrm{g}$ & $13.9 \mathrm{a}-\mathrm{g}$ & $16.5 \mathrm{c}-\mathrm{k}$ \\
\hline 44 & Ak-702 & $19.9 \mathrm{a}-\mathrm{g}$ & 16.7 a-d & 18.4 a-f & $18.5 b-h$ & 14.6 a-e & $16.6 \mathrm{c}-\mathrm{j}$ \\
\hline 45 & Ak Buğday & $19.2 \mathrm{~b}-\mathrm{h}$ & $16.1 \mathrm{a}-\mathrm{j}$ & $17.7 \mathrm{~b}-\mathrm{k}$ & 20.0 a-f & $14.0 \mathrm{a}-\mathrm{g}$ & 17.0 b-I \\
\hline 46 & Ankara 093/44 & $18.7 \mathrm{c}-\mathrm{j}$ & $15.5 \mathrm{a}-\mathrm{I}$ & 17.2 b-n & $18.3 \mathrm{~b}-\mathrm{j}$ & $14.1 \mathrm{a}-\mathrm{g}$ & $16.3 \mathrm{~d}-\mathrm{m}$ \\
\hline 47 & Conkesme & 20.8 a-f & $16.3 \mathrm{a}-\mathrm{g}$ & 18.6 a-e & $21.4 a b$ & $15.0 \mathrm{abc}$ & $18.2 \mathrm{abc}$ \\
\hline 48 & Haymana 79 & $18.2 \mathrm{~d}-\mathrm{m}$ & 15.4 a-I & $16.9 \mathrm{~d}-\mathrm{o}$ & $18.0 \mathrm{c}-\mathrm{j}$ & $13.5 \mathrm{a}-\mathrm{h}$ & $15.8 \mathrm{f}-\mathrm{o}$ \\
\hline 49 & Hawk (Şahin) & $17.2 \mathrm{~g}-\mathrm{o}$ & $14.3 c-n$ & $15.6 \mathrm{l}-\mathrm{r}$ & $18.2 \mathrm{~b}-\mathrm{j}$ & $14.9 \mathrm{abc}$ & $16.6 \mathrm{c}-\mathrm{j}$ \\
\hline 50 & Kılçıksız Buğday & 19.7 a-h & 14.0 e-o & $16.8 \mathrm{e}-\mathrm{o}$ & $17.9 \mathrm{c}-\mathrm{k}$ & 13.4 b-h & $15.5 \mathrm{~h}-\mathrm{q}$ \\
\hline 51 & Kırkpınar 79 & $19.3 b-h$ & 15.6 a-I & 17.4 b-I & 19.9 a-f & 14.4 a-f & $17.1 \mathrm{~b}-\mathrm{I}$ \\
\hline 52 & Kırmızı Kılçık & $19.9 \mathrm{a}-\mathrm{g}$ & $15.7 \mathrm{a}-\mathrm{k}$ & $17.8 \mathrm{~b}-\mathrm{j}$ & $18.8 b-h$ & $14.1 \mathrm{a}-\mathrm{g}$ & $16.5 \mathrm{c}-\mathrm{I}$ \\
\hline 53 & Kırmızı Yerli & $20.2 a-g$ & 16.6 a-e & $18.3 \mathrm{a}-\mathrm{g}$ & 20.8 a-e & 14.5 a-e & $17.6 \mathrm{a}-\mathrm{g}$ \\
\hline 54 & Koca Buğday & $20.2 a-g$ & 16.5 a-e & $18.3 a-g$ & $17.6 \mathrm{e}-\mathrm{k}$ & $15.1 \mathrm{abc}$ & $16.4 \mathrm{c}-\mathrm{I}$ \\
\hline 55 & Köse 220/39 & $20.3 a-g$ & $16.3 \mathrm{a}-\mathrm{g}$ & $18.3 \mathrm{a}-\mathrm{g}$ & 19.7 a-f & $14.0 \mathrm{a}-\mathrm{g}$ & $16.8 \mathrm{c}$-I \\
\hline 56 & Özlü Buğday & 20.7 a-f & $16.9 \mathrm{abc}$ & $18.8 \mathrm{abc}$ & 19.8 a-f & $13.0 \mathrm{c}-\mathrm{I}$ & $16.4 \mathrm{c}-\mathrm{I}$ \\
\hline 57 & Polatlı Kösesi & 20.8 a-f & $14.9 \mathrm{a}-\mathrm{m}$ & $17.8 \mathrm{~b}-\mathrm{k}$ & $21.1 \mathrm{abc}$ & $13.9 \mathrm{a}-\mathrm{g}$ & $17.3 \mathrm{~b}-\mathrm{h}$ \\
\hline 58 & Sert Buğday & $21.2 \mathrm{a}-\mathrm{d}$ & 15.5 a-I & 18.4 a-f & $18.2 \mathrm{~b}-\mathrm{j}$ & $13.8 \mathrm{a}-\mathrm{g}$ & $15.8 \mathrm{~g}-\mathrm{o}$ \\
\hline 59 & Sürak 1593/51 & 20.8 a-f & 15.2 a-l & $18.1 \mathrm{a}$ & 20.8 a-e & $15.8 \mathrm{a}$ & $18.2 \mathrm{abc}$ \\
\hline 60 & Tir & 19.4 b-h & $17.3 \mathrm{a}$ & 18.4 a-f & $18.2 \mathrm{~b}-\mathrm{j}$ & $15.0 \mathrm{abc}$ & $16.5 \mathrm{c}-\mathrm{k}$ \\
\hline 61 & Yayla 305 & $19.3 \mathrm{~b}-\mathrm{h}$ & $17.2 \mathrm{ab}$ & $18.2 \mathrm{a}-\mathrm{h}$ & $18.2 \mathrm{~b}-\mathrm{j}$ & $15.0 \mathrm{abc}$ & $16.4 \mathrm{c}-\mathrm{I}$ \\
\hline 62 & Zerin & $17.7 \mathrm{e}-\mathrm{o}$ & $16.1 \mathrm{a}-\mathrm{j}$ & $16.9 \mathrm{c}-\mathrm{O}$ & $18.0 \mathrm{c}-\mathrm{j}$ & $14.0 \mathrm{a}-\mathrm{g}$ & 15.9 e-n \\
\hline 63 & Bezostaja 1 & $17.4 \mathrm{~g}-\mathrm{o}$ & $15.9 \mathrm{a}-\mathrm{k}$ & $16.7 \mathrm{f}-\mathrm{p}$ & $17.6 \mathrm{e}-\mathrm{I}$ & $14.4 \mathrm{a}-\mathrm{f}$ & $15.8 \mathrm{f}-\mathrm{o}$ \\
\hline 64 & Karasu 90 & $19.2 \mathrm{~b}-\mathrm{h}$ & 16.5 a-e & $17.9 \mathrm{~b}-\mathrm{I}$ & $19.2 \mathrm{~b}-\mathrm{g}$ & $14.0 \mathrm{a}-\mathrm{g}$ & $16.5 \mathrm{c}-\mathrm{I}$ \\
\hline \multicolumn{2}{|c|}{ Ortalama } & 18.6 & 14.8 & 16.7 & 18.4 & 13.7 & 16.0 \\
\hline \multicolumn{2}{|c|}{ P değeri (Genotip) } & 0.000 & 0.000 & 0.000 & 0.000 & 0.000 & 0.000 \\
\hline \multicolumn{2}{|c|}{$\mathrm{P}$ değeri (Yıl) } & - & - & 0.000 & - & - & 0.000 \\
\hline \multicolumn{2}{|c|}{ P değeri (Genotip x Yıl) } & - & - & 0.000 & - & - & 0.000 \\
\hline AÖ & (Genotip) & 2.6 & 2.2 & 1.5 & 2.7 & 1.8 & 1.5 \\
\hline Var & syon katsayısı (\%) & 5.27 & 5.48 & 4.95 & 5.41 & 5.03 & 5.02 \\
\hline$S \bar{x}$ & & 0.69 & 0.58 & 0.41 & 0.70 & 0.49 & 0.40 \\
\hline Lati & etkinliği (\% ) & 197.7 & 153.8 & & 134.5 & 103.4 & \\
\hline
\end{tabular}



Genotypes"

Table 2. Buğday genotiplerinin tane dolum süreleri (gün)

Table 1. Grain filling periods of wheat genotypes (days)

\begin{tabular}{|c|c|c|c|c|c|c|c|}
\hline \multirow{2}{*}{ No } & \multirow{2}{*}{ Genotipler } & \multicolumn{3}{|c|}{ Sulu koşullar } & \multicolumn{3}{|c|}{ Geç kuraklık } \\
\hline & & $2011-12$ & $2012-13$ & Birleşik & $2011-12$ & $2012-13$ & Birleşik \\
\hline 1 & Aksel 2000 & $39.5 \mathrm{hi}^{*}$ & 33.4 a-f & $36.1 \mathrm{~d}-\mathrm{j}$ & $33.1 \mathrm{c}-\mathrm{g}$ & $26.5 \mathrm{a}-\mathrm{k}$ & 29.8 a-I \\
\hline 2 & Aldane & $41.1 \mathrm{~b}-\mathrm{i}$ & 33.4 a-f & $37.2 \mathrm{~b}-\mathrm{j}$ & 31.9 efg & 27.4 a-g & I-I \\
\hline 3 & Alparslan & 39.4 hi & 32.4 a-I & $35.8 \mathrm{f}-\mathrm{j}$ & $35.8 \mathrm{~b}-\mathrm{e}$ & 27.8 a-f & 30.9 a-f \\
\hline 4 & Altay 2000 & 39.6 ghi & 32.1 a-ו & $35.8 \mathrm{f}-\mathrm{j}$ & $34.7 \mathrm{~b}-\mathrm{g}$ & $25.7 \mathrm{~b}-\mathrm{m}$ & 29.7 a-I \\
\hline 5 & Atlı 2002 & 46.3 a-i & 32.9 a-h & 39.4 a-h & $34.3 \mathrm{~b}-\mathrm{g}$ & 25.2 b-p & ו- 29.8 a- \\
\hline 6 & Aytın 98 & $41.5 \mathrm{a}-\mathrm{i}$ & 28.9 hı & $35.1 \mathrm{lj}$ & $33.0 \mathrm{c}-\mathrm{g}$ & $24.3 \mathrm{~d}-\mathrm{t}$ & 29.5 a-I \\
\hline 7 & Bağcı 2002 & $44.3 \mathrm{a}-\mathrm{i}$ & 32.5 a-h & 38.4 a-j & $34.3 b-g$ & $25.1 b-q$ & $29.0 \mathrm{c}-\mathrm{I}$ \\
\hline 8 & Bayraktar 2000 & $48.8 \mathrm{ab}$ & 31.4 a-ı & $40.4 \mathrm{abc}$ & $37.6 \mathrm{ab}$ & $26.0 \mathrm{~b}-\mathrm{I}$ & 31.0 a-f \\
\hline 9 & Bolal 2973 & $41.5 \mathrm{a}-\mathrm{i}$ & 33.5 a-f & $37.2 \mathrm{~b}-\mathrm{j}$ & 35.4 b-f & 25.6 b-n & 27.8 e-I \\
\hline 10 & Bereket & $40.1 \mathrm{e}-\mathrm{i}$ & 31.1 a-I & $35.5 \mathrm{~g}-\mathrm{j}$ & 31.7 efg & $25.5 \mathrm{~b}-\mathrm{o}$ & $28.3 \mathrm{~d}-\mathrm{I}$ \\
\hline 11 & Çetinel 2000 & 41.4 a-i & 31.0 a-ı & $36.1 \mathrm{~d}-\mathrm{j}$ & $32.6 \mathrm{efg}$ & $24.3 \mathrm{~d}-\mathrm{t}$ & 28.6 c-I \\
\hline 12 & Dağdaş 94 & $40.1 \mathrm{f}-\mathrm{i}$ & $34.7 \mathrm{abc}$ & $37.1 \mathrm{c}-\mathrm{j}$ & $34.5 b-g$ & $29.8 \mathrm{a}$ & 31.1 a-e \\
\hline 13 & Demir 2000 & 44.8 a-i & 32.0 a-ı & $38.2 \mathrm{a-j}$ & 31.6 efg & 25.2 b-p & 30.0 a-I \\
\hline 14 & Doğu 88 & $44.0 \mathrm{a}-\mathrm{i}$ & $29.0 \mathrm{gh}$ & $38.0 \mathrm{a-j}$ & $33.8 \mathrm{~b}-\mathrm{g}$ & $21.9 \mathrm{~m}-\mathrm{v}$ & 27.2 I \\
\hline 15 & ES 26 & $44.3 \mathrm{a}-\mathrm{i}$ & 32.6 a-h & $38.4 a-j$ & $35.6 b-f$ & $27.2 \mathrm{a}-\mathrm{h}$ & 29.2 a-I \\
\hline 16 & Gerek 79 & $45.3 \mathrm{a}-\mathrm{i}$ & 32.6 a-h & 39.2 a-I & $34.9 \mathrm{~b}-\mathrm{g}$ & 27.1 a-ı & 30.9 a-f \\
\hline 17 & Gün 91 & $40.4 \mathrm{~d}-\mathrm{i}$ & 31.3 a-ı & 35.4 hij & $31.3 \mathrm{fg}$ & 26.6 a-j & 29.9 a-I \\
\hline 18 & Harmankaya 99 & $41.5 \mathrm{a}-\mathrm{i}$ & $30.3 \mathrm{~d}-\mathrm{I}$ & $35.8 \mathrm{e}-\mathrm{j}$ & $32.1 \mathrm{efg}$ & $24.5 \mathrm{c}-\mathrm{t}$ & 29.7 a-I \\
\hline 19 & İkizce 96 & 47.8 a-e & 31.8 a-ı & $39.5 \mathrm{a}-\mathrm{g}$ & $37.0 \mathrm{a}-\mathrm{d}$ & $23.4 \mathrm{~h}-\mathrm{v}$ & $28.7 \mathrm{c}-\mathrm{I}$ \\
\hline 20 & İzgi 2001 & 42.0 a-i & $31.0 \mathrm{~b}-\mathrm{I}$ & $36.1 \mathrm{~d}-\mathrm{j}$ & 31.9 efg & $24.3 \mathrm{~d}-\mathrm{t}$ & $27.9 \mathrm{~d}-\mathrm{I}$ \\
\hline 21 & Karahan 99 & $43.2 \mathrm{a}-\mathrm{i}$ & $32.8 \mathrm{a}-\mathrm{h}$ & $37.6 \mathrm{a-j}$ & $33.5 \mathrm{~b}-\mathrm{g}$ & $25.8 \mathrm{~b}-\mathrm{I}$ & $28.6 \mathrm{c}-\mathrm{I}$ \\
\hline 22 & Kate A-1 & 45.4 a-i & $30.8 \mathrm{c}-1$ & $37.9 \mathrm{a-j}$ & $34.2 \mathrm{~b}-\mathrm{g}$ & $23.9 \mathrm{f}-\mathrm{u}$ & 28.0 d-I \\
\hline 23 & Kıraç 66 & $45.7 \mathrm{a}-\mathrm{i}$ & 31.4 a-ı & $38.5 \mathrm{a}-\mathrm{j}$ & $33.0 \mathrm{c}-\mathrm{g}$ & $25.2 b-p$ & 29.0 b-I \\
\hline 24 & Kırgiz 95 & $45.1 \mathrm{a}-\mathrm{i}$ & $33.8 \mathrm{a}-\mathrm{d}$ & $39.6 a-g$ & $34.8 \mathrm{~b}-\mathrm{g}$ & $24.6 \mathrm{c}-\mathrm{t}$ & $28.3 \mathrm{~d}-\mathrm{I}$ \\
\hline 25 & Kırik & $41.2 \mathrm{~b}-\mathrm{i}$ & 31.4 a-ı & $35.7 \mathrm{f}-\mathrm{j}$ & $32.9 \mathrm{~d}-\mathrm{g}$ & $23.5 \mathrm{~g}-\mathrm{v}$ & 29.1 b-I \\
\hline 26 & Kutluk 94 & 44.9 a-i & $29.5 \mathrm{f}-\mathrm{I}$ & $36.9 \mathrm{c}-\mathrm{j}$ & $31.2 \mathrm{fg}$ & 27.9 a-e & $30.8 \mathrm{a}-\mathrm{g}$ \\
\hline 27 & Lancer & $43.7 \mathrm{a}-\mathrm{i}$ & $33.0 \mathrm{a}-\mathrm{g}$ & $37.9 \mathrm{a-j}$ & $33.1 \mathrm{c}-\mathrm{g}$ & $25.3 b-p$ & 30.4 a-I \\
\hline 28 & Mızrak & $39.3 \mathrm{i}$ & 33.6 a-e & $36.1 \mathrm{~d}-\mathrm{j}$ & $35.0 \mathrm{~b}-\mathrm{f}$ & $28.2 \mathrm{abc}$ & $32.4 a b$ \\
\hline 29 & Müfitbey & 42.0 a-i & 32.9 a-h & $37.2 \mathrm{c}-\mathrm{j}$ & $33.6 \mathrm{~b}-\mathrm{g}$ & 27.7 a-f & 30.5 a-I \\
\hline 30 & Nacibey & $43.3 \mathrm{a}-\mathrm{i}$ & 29.9 d-ו & $36.4 \mathrm{c}-\mathrm{j}$ & $33.3 \mathrm{~b}-\mathrm{g}$ & $24.8 \mathrm{c}-\mathrm{r}$ & 29.4 a-I \\
\hline 31 & Nenehatun & 44.0 a-i & 32.5 a-h & $38.2 \mathrm{a}-\mathrm{j}$ & $35.6 b-f$ & $21.6 \mathrm{o}-\mathrm{v}$ & $27.4 \mathrm{ghı}$ \\
\hline 32 & Palandöken 97 & 47.5 a-f & $35.0 a b$ & $41.4 \mathrm{a}$ & $39.9 \mathrm{a}$ & $26.5 \mathrm{a}-\mathrm{k}$ & $31.9 \mathrm{abc}$ \\
\hline 33 & Pehlivan & $39.5 \mathrm{hi}$ & 31.0 a-I & $34.9 \mathrm{j}$ & $34.0 \mathrm{~b}-\mathrm{g}$ & $24.4 c-t$ & 29.1 b-I \\
\hline 34 & Prostor & 42.6 a-i & $35.1 \mathrm{a}$ & $38.8 \mathrm{a}-\mathrm{j}$ & $33.8 \mathrm{~b}-\mathrm{g}$ & $25.3 b-p$ & 29.7 a- \\
\hline 35 & Soyer02 & 46.9 a-i & 30.6 d-I & $38.7 \mathrm{a}-\mathrm{j}$ & $34.7 \mathrm{~b}-\mathrm{g}$ & $21.2 r-v$ & 27.7 e-I \\
\hline 36 & Sönmez 2001 & $40.6 \mathrm{c}-\mathrm{i}$ & $33.2 \mathrm{a}-\mathrm{f}$ & $36.8 \mathrm{c}-\mathrm{j}$ & $35.6 b-f$ & $25.6 \mathrm{~b}-\mathrm{m}$ & 29.4 a-I \\
\hline 37 & Sultan 95 & $40.8 c-i$ & $35.1 \mathrm{a}$ & $37.8 a-j$ & $32.2 \mathrm{efg}$ & 27.6 a-f & $31.3 \mathrm{a}-\mathrm{d}$ \\
\hline 38 & Süzen 97 & 48.0 a-d & 32.6 a-h & $40.3 \mathrm{abc}$ & $34.4 \mathrm{~b}-\mathrm{g}$ & $23.2 \mathrm{I}-\mathrm{V}$ & 29.0 b-I \\
\hline 39 & Tosunbey & $41.3 \mathrm{~b}-\mathrm{i}$ & 32.7 a-h & $37.1 \mathrm{c}-\mathrm{j}$ & $35.1 b-f$ & $28.0 \mathrm{a}-\mathrm{d}$ & 30.8 a-h \\
\hline 40 & Türkmen & $43.2 \mathrm{a}-\mathrm{i}$ & 33.1 a-f & $38.5 a-j$ & $35.0 \mathrm{~b}-\mathrm{g}$ & $28.9 a b$ & $32.5 \mathrm{a}$ \\
\hline 41 & Uzunyayla & $44.8 \mathrm{a}-\mathrm{i}$ & 31.6 a-I & $38.3 \mathrm{a}-\mathrm{j}$ & $32.8 \mathrm{~d}-\mathrm{g}$ & $23.1 \mathrm{j}-\mathrm{v}$ & $28.3 \mathrm{~d}-\mathrm{I}$ \\
\hline 42 & Yakar 99 & 41.9 a-i & 30.7 c-I & $36.8 \mathrm{c}-\mathrm{j}$ & $30.6 \mathrm{~g}$ & $23.5 \mathrm{~h}-\mathrm{v}$ & $28.6 \mathrm{c}-\mathrm{I}$ \\
\hline 43 & Zencirci 2002 & $44.2 \mathrm{a}-\mathrm{i}$ & $33.2 \mathrm{a}-\mathrm{f}$ & $38.9 \mathrm{a}-\mathrm{j}$ & $32.5 \mathrm{efg}$ & $24.9 \mathrm{c}-\mathrm{r}$ & 29.4 a-ı \\
\hline 44 & Ak-702 & 43.4 a-i & 31.8 a-ı & $37.8 \mathrm{a}-\mathrm{j}$ & $33.9 \mathrm{~b}-\mathrm{g}$ & $25.3 b-p$ & 29.9 a-I \\
\hline 45 & Ak Buğday & 43.6 a-i & 33.7 a-e & $38.8 \mathrm{a}-\mathrm{j}$ & $32.0 \mathrm{efg}$ & $24.8 \mathrm{c}-\mathrm{s}$ & $28.8 \mathrm{c}-\mathrm{I}$ \\
\hline 46 & Ankara 093/44 & 47.8 a-d & 31.1 a-ı & 39.9 a-e & $32.7 \mathrm{~d}-\mathrm{g}$ & $22.4 \mathrm{l}-\mathrm{V}$ & 27.7 e-I \\
\hline 47 & Conkesme & 46.1 a-i & $33.3 \mathrm{a}-\mathrm{f}$ & $40.1 \mathrm{a}-\mathrm{d}$ & 32.0 efg & $25.2 b-p$ & $28.4 \mathrm{~d}-\mathrm{I}$ \\
\hline 48 & Haymana 79 & 46.5 a-i & $34.7 \mathrm{abc}$ & $41.2 \mathrm{ab}$ & $34.3 b-g$ & $26.1 \mathrm{a}-\mathrm{I}$ & 29.4 a-ı \\
\hline 49 & Hawk (Şahin) & 47.7 a-f & 31.9 a-ı & $39.5 a-g$ & $35.4 b-f$ & $25.6 b-n$ & 30.2 a-ı \\
\hline 50 & Kılçıksız Buğday & 46.8 a-i & 31.4 a-I & 39.2 a-h & $33.2 \mathrm{c}-\mathrm{g}$ & $21.0 \mathrm{~s}-\mathrm{v}$ & 27.9 d-ו \\
\hline 51 & Kırkpınar 79 & $48.1 \mathrm{abc}$ & 28.4 I & $38.4 a-j$ & $34.1 \mathrm{~b}-\mathrm{g}$ & 20.8 tuv & 27.2 I \\
\hline 52 & Kırmızı Kılçık & $45.3 \mathrm{a-i}$ & I1.1 a- & $38.1 \mathrm{a}-\mathrm{j}$ & $35.0 \mathrm{~b}-\mathrm{f}$ & $21.3 q-v$ & 29.0 c-I \\
\hline 53 & Kırmızı Yerli & $45.0 \mathrm{a}-\mathrm{i}$ & I-I & $38.4 a-j$ & $31.6 \mathrm{efg}$ & $21.7 n-v$ & 28.6 c-I \\
\hline 54 & Koca Buğday & $46.2 \mathrm{a}-\mathrm{i}$ & 31.9 ו-ו & $39.2 a-h$ & $33.8 \mathrm{~b}-\mathrm{g}$ & 20.4 uv & 27.5 f-I \\
\hline 55 & Köse 220/39 & 46.5 a-i & $32.5 a-h$ & 39.7 a-f & $34.1 \mathrm{~b}-\mathrm{g}$ & $22.7 \mathrm{k}-\mathrm{v}$ & $27.8 \mathrm{e}-1$ \\
\hline 56 & Özlü Buğday & $46.9 a-i$ & $32.9 \mathrm{a}-\mathrm{h}$ & $40.4 \mathrm{abc}$ & $32.9 \mathrm{c}-\mathrm{g}$ & $24.1 \mathrm{e}-\mathrm{u}$ & ו- ו- \\
\hline 57 & Polatlı Kösesi & 44.9 a-i & 30.0 d-ו & $37.3 \mathrm{~b}-\mathrm{j}$ & 31.6 efg & $19.8 v$ & $27.3 \mathrm{hl}$ \\
\hline 58 & Sert Buğday & $46.0 \mathrm{a}-\mathrm{i}$ & 30.0 d-I & $38.2 \mathrm{a-j}$ & $33.4 \mathrm{~b}-\mathrm{g}$ & $21.60-v$ & 29.6 a-I \\
\hline 59 & Sürak $1593 / 51$ & $45.3 \mathrm{a}-\mathrm{i}$ & 32.0 a-I & $38.8 \mathrm{a-j}$ & $32.8 \mathrm{~d}-\mathrm{g}$ & $20.0 \mathrm{v}$ & 27.7 e-I \\
\hline 60 & Tir & 47.0 a-h & ו ו- e. & $38.4 a-j$ & $35.2 b-f$ & 20.5 uv & 27.7 e-I \\
\hline 61 & Yayla 305 & $45.7 \mathrm{a}-\mathrm{i}$ & $30.5 \mathrm{~d}-\mathrm{I}$ & $38.1 \mathrm{a}-\mathrm{j}$ & $33.8 \mathrm{~b}-\mathrm{g}$ & 20.9 tuv & 27.8 e- I \\
\hline 62 & Zerin & $48.9 \mathrm{a}$ & $30.5 \mathrm{~d}-\mathrm{I}$ & 39.9 a-e & $34.0 \mathrm{~b}-\mathrm{g}$ & $21.5 p-v$ & 27.7 e-I \\
\hline 63 & Bezostaja 1 & $47.2 \mathrm{a}-\mathrm{g}$ & $31.1 \mathrm{a}-\mathrm{I}$ & 39.4 a-h & $37.3 \mathrm{abc}$ & $22.4 \mathrm{l}-\mathrm{v}$ & 28.0 d-I \\
\hline 64 & Karasu 90 & $48.7 \mathrm{ab}$ & 31.0 a-I & $40.4 a b c$ & $34.1 \mathrm{~b}-\mathrm{g}$ & $21.7 n-v$ & 28.7 C-I \\
\hline \multicolumn{2}{|c|}{ Ortalama } & 44.2 & 31.9 & 38.1 & 33.8 & 24.4 & 29.1 \\
\hline \multicolumn{2}{|c|}{ P değeri (Genotip) } & 0.000 & 0.000 & 0.000 & 0.000 & 0.000 & 0.000 \\
\hline \multicolumn{2}{|c|}{$P$ değeri $(Y, I)$} & - & - & 0.000 & - & - & 0.000 \\
\hline \multicolumn{2}{|c|}{ P değeri (Genotip x Yıl) } & - & - & 0.000 & - & - & 0.000 \\
\hline AÖ & Genotip) & 6.2 & 3.3 & 3.2 & 3.5 & 3.2 & 2.7 \\
\hline Var & yon katsayısı (\% ) & 5.19 & 3.84 & 4.58 & 3.87 & 4.81 & 5.05 \\
\hline$S \bar{x}$ & & 1.62 & 0.87 & 0.87 & 0.93 & 0.83 & 0.74 \\
\hline Lati & tkinliği (\% ) & 123.2 & 102.0 & & 103.4 & 114.2 & \\
\hline
\end{tabular}

* Aynı harf ile işaretli ortalamalar birbirinden farksızdır.* Averages followed by same letter are not statistically different. 
İki denemede de, daha düşük toprak nem içeriği nedeniyle tane dolum süresi ikinci yılda birinci yıla göre önemli derecede kısalmıştır. Ürün yılları ve genotipler ortalamasına göre, tane dolum süresi sulu koşullarda 38.1 gün iken, geç kuraklık stresi koşullarında toprak nem içeriğindeki azalmaya bağlı olarak kısalmış ve 29.1 gün olmuştur. Topraktaki nem yetersizliği veya kuraklığın, yeşil dokularda yaşlanma ve kurumayı hızlandırarak tane dolum süresini kısalttığı bilinmektedir (Öztürk 1999).

Yılların ortalamalarına göre, sulu koşullar ile karşılaştııılığında geç kuraklık stresi koşullarında tane dolum süresinin en az (3.76.1 gün) Mızrak, Alparslan, Gün 91, Aytın 98, Pehlivan, Dağdaş 94, Türkmen, Altay 2000, Harmankaya 99 ve Kutluk 94 genotiplerinde; en fazla (12.6-11.7 gün) ise Özlü Buğday, Ankara 193/44, Zerin, Köse 220/39, Haymana 79, Conkesme ve Koca Buğday genotiplerinde kısaldığı tespit edilmiştir. Tane dolum süresi en az kısalan genotiplerin son dönemlerde geliştirilen ıslah çeşitlerinden, en fazla kısalan genotiplerin ise yerel genotipler veya eski çeşitlerden olması dikkat çekmiştir.

\section{Sonuç}

Bulgular, vejetatif dönem ve tane dolum süresinin genotipe ve çevre koşullarına bağlı olduğunu, geç kuraklık stresinin esas olarak tane dolum süresini kısalttığını göstermiştir. İki denemede de, vejetatif dönem ile tane verimi arasındaki ilişki önemsiz olmuştur (sulu koşullar ve geç kuraklık koşullarında sırasıyla $r=-0.088$ ve $r=-0.107$ ). Sulu koşullarda tane dolum süresi ile tane verimi arasındaki ilişki de önemsiz bulunmuştur ( $r=-0.020)$. Geç kuraklık koşullarında, tane dolum süresi ile başaktaki tane sayısı $(r=0.189)$ ve bin tane ağırlığı $(r=0.213)$ arasındaki nispeten yüksek ve önemsiz ilişkiler, tane dolum süresi ile tane verimi arasında olumlu ve önemli bir ilişkinin $\left(r=0.281^{*}\right)$ çıkmasını sağlamıştır.

\section{Kaynaklar}

Bruckner P.L. and Frohberg R.C., 1987. Rate and duration of grain fill in spring wheat. Crop Sci., 27(3): 451-455

Çağlar Ö., Öztürk A. ve Bulut S., 2006. Bazı ekmeklik buğday çeşitlerinin erzurum ovası koşullarına adaptasyonu. Atatürk Üni. Ziraat Fak. Dergisi, 37(1): 1-7

Egli D.B., 1998. Seed Biology and the Yield of Grain Crops. CAB International, Oxford.
Farooq M., Hussain M. and Siddique K.H., 2014. Drought stress in wheat during flowering and grain-filling periods. Critical Reviews in Plant Sciences, 33(4): 331-349

Fischer R.A., Maurer R., 1976. Crop temperature modification and yield potential in a dwarf spring wheat. Crop Science, 16(6): 855-859

Gebeyehou G., Knott O.R. and Baker R.J., 1982. Relationship among duration of vegetative and grain filling phases, yield components and grain yield in durum wheat cultivars. Crop Sci., 22(2): 287-290

Hunt L.A., Van der Porten G. and Pararajasingham S., 1991. Postanthesis Temperature Effects on Duration and Rate of Grain Filling in Some Winter And Spring Wheats. Can. J. Plant Sci., 71 (3): 609-617

Nass H.G. and Raiser B., 1975. Grain filling period and grain yield relationships in spring wheat. Can. J. Plant Sci., 55(3): 673-678

Nicolas M.E., Gleadow R.M. and Dalling M.J., 1985. Effect of post-anthesis drought on cell division and starch accumulation in developing wheat grains. Ann. Bot., 55(3): 433-444

Öztürk A. ve Akkaya A., 1996. Kışlık buğday genotiplerinde (Triticum aestivum L.) tane verimi. verim unsurları ve fenolojik dönemler üzerine bir araştırma. Atatürk Üniv. Ziraat Fak. Derg., 27(2): 187-202

Öztürk A. ve Akten Ş., 1999. Kışlık buğdayda bazı morfofizyolojik karakterler ve tane verimine etkileri. Tr. J. of Agriculture and Forestry, 23 (Ek Sayı 2): 409-422

Öztürk A. ve Çağlar Ö., 1999. Kışlık buğdayda kuraklığın vejatatif dönem, tane dolum dönemi ve tane dolum oranına etkisi. Atatürk Üni. Ziraat Fak. Dergisi, 30(1): 1-10.

Öztürk A., 1999. Kuraklığın kışlık buğdayın gelişmesi ve verimine etkisi. Turk J. Agric. For., 23(5): 531-540

Prasad P., Pisipati S., Momčilović I. and Ristic Z., 2011. Independent and combined effects of high temperature and drought stress during grain filling on plant yield and chloroplast efDtu expression in spring wheat. Journal of Agronomy and Crop Science, 197: 430-441.

Sanjari Pireivatlou A.G., Aliyev R.T. and Sorkhi Lalehloo B., 2011. Grain filling rate and duration in bread wheat under Irrigated and drought stressed conditions. Journal of Plant Physiology and Breeding, 1(1): 75-92

Tiwari V., 2007. Grain filling duration as a means for Increasing yield in spring wheat. Indian $\mathrm{J}$. Genet., 67(4): 365-368 
Aydın and Öztürk "Effect of Late Drought on Vegetative Period and Grain Filling Period in Bread Wheat Genotypes"

Wahid A., Gelani S., Ashraf M. and Foolad M.R., Wiegand C.L., Gebermann A.H. and Cuellar J.A., 2007. Heat Tolerance in Plants: An Overview. Environmental and Experimental Botany, 61(3): 199-223.

1981. Development and yield of hard red winter wheats under semitropical conditions. Agron. J., 73(1): 29-37 